\title{
Prognostic implications of cimetidine on advanced serous ovarian carcinoma related to cyclooxygenase- 2 expression
}

\author{
KENJI NIWA $^{1}$, KOKYO ONOGI $^{1}$, YUN WU $^{1}$, HIDEKI MORI $^{2}$, YOSUKE INOUE $^{3}$ and TERUHIKO TAMAYA ${ }^{1}$ \\ Departments of ${ }^{1}$ Obstetrics and Gynecology, and ${ }^{2}$ Tumor Pathology, Gifu University Graduate School of Medicine, \\ Gifu 501-1194; ${ }^{3}$ Dainippon-Sumitomo Pharma Co., Chiba, Japan
}

Received October 29, 2007; Accepted November 29, 2007

\begin{abstract}
This study was performed to identify the contribution of cimetidine to chemotherapy for epithelial ovarian carcinoma. Cimetidine was administered two weeks before surgery in combination with platinum-based chemotherapy, and the treatment was continued for two years. Cyclooxygenase-2 (COX-2) expression was also evaluated. The chemotherapy regimens did not affect patient prognosis. The effect of cimetidine was more marked in patients who showed overexpression of COX-2. Platinum-based chemotherapy combined with cimetidine, as a first-line therapy, may improve the prognosis of patients with advanced serous ovarian carcinoma.
\end{abstract}

\section{Introduction}

Ovarian carcinoma has the highest mortality rate among gynecological malignancies. The prognosis for patients with ovarian carcinoma depends on the stage of the disease at diagnosis, as well as on age, histologic type and tumor grade (1). Most women with advanced ovarian cancer are offered some form of chemotherapy after surgery, but the 5-year survival rate after diagnosis is assumed to be $\sim 30 \%$ (2). Paclitaxel has recently been introduced to the treatment for ovarian cancer; however, the superiority of chemotherapy combined with paclitaxel to conventional chemotherapy [cyclophosphamide, doxorubicin and cisplatin (CAP) or doxorubicin and cisplatin (AP)] has yet to be demonstrated $(3,4)$. Cimetidine is a histamine $\mathrm{H}_{2}$-receptor antagonist that has been shown to inhibit tumor growth in nude mice bearing tumors produced by ovarian cancer cell lines (5) and to improve the prognosis in patients with colorectal cancer (6).

Correspondence to: Dr Kenji Niwa, Department of Obstetrics and Gynecology, Gifu University Graduate School of Medicine, 1-1 Yanagido, Gifu 501-1194, Japan

E-mail: kniwa@gifu-u.ac.jp

Abbreviations: COX-2, cyclooxygenase-2; RFS, refractory free survival; OS, overall survival

Key words: cimetidine, ovarian cancer, survival, cyclooxygenase-2
Histamine is reported to exert both proliferative and angiogenic effects via $\mathrm{H}_{2} / \mathrm{H}_{4}$ receptor activation (6). These effects are mediated by cyclooxygenase-2 (COX-2)-related PGE2 production in COX-2-expressing cancer cells (7). COX-2 is also overexpressed in the vast majority of colorectal cancers, as well as in other solid tumors including ovarian cancer (8), and COX-2 expression is regarded as a clinicopathological parameter of aggressiveness and of an unfavorable prognosis $(8,9)$. Cimetidine prevents metastasis by blocking E-selectin expression (10) and by inhibiting histamine-induced angiogenesis (11). We thus investigated whether the administration of cimetidine affected the survival of patients whose ovarian cancer overexpressed COX-2.

The principle aim of this study was to clarify the clinical implications of cimetidine, particularly in response to treatment and clinical outcome, in a single-institution series of patients with untreated primary ovarian cancer.

\section{Materials and methods}

Patients. The clinical records of 28 patients with advanced serous cystadenocarcinoma ovarian cancer (FIGO stage III/IV), who were admitted, treated and monitored at the Department of Obstetrics and Gynecology of Gifu University Hospital between January 1993 and December 2001, were evaluated for clinical prognostic factors (patient age, tumor stage), survival status and cause of death (cancer-associated, cancerindependent and undetermined). The clinicopathological characteristics are listed in Table I. The mean age of patients at diagnosis was $55.1 \pm 12.2$ years. The median following-up period was $3.1 \pm 1.4$ years.

Standard surgical intervention consisted of bilateral salpingo-oophorectomy, total abdominal hysterectomy and partial omentectomy. Para-aortic and pelvic lymphadenectomy and cytology of ascites or peritoneal washing cytology were also routinely performed. In addition, all patients underwent tumor reductive surgery and were sub-optimally debulked.

Pathological study. Histologic type and grade were determined by two of the authors (K.N. and H.M.) using the previouslydescribed WHO classification (12). Patients with serous tumors were included in this study, while those with benign lesions, metastatic ovarian tumors or borderline tumors were excluded. Eighteen patients (64.3\%) were in stage III and 10 $(35.7 \%)$ were in stage IV. 
Table I. Patient characteristics.

\begin{tabular}{|c|c|c|c|c|}
\hline & Total & Cimetidine group & Control group & $\mathrm{P}$-value \\
\hline Number of patients & 28 & 11 & 17 & \\
\hline Mean age at diagnosis & $55.1 \pm 12.2$ & $56.0 \pm 8.6$ & $54.5 \pm 14.3$ & 0.2501 \\
\hline \multicolumn{5}{|l|}{ Tumor grade } \\
\hline 1 & 12 & 6 & 6 & 0.5390 \\
\hline 2 & 11 & 3 & 8 & 0.5151 \\
\hline 3 & 5 & 2 & 3 & 0.9712 \\
\hline \multicolumn{5}{|l|}{ Histologic type } \\
\hline Serous & 28 & 11 & 17 & \\
\hline \multicolumn{5}{|l|}{ FIGO stage } \\
\hline III & 18 & 5 & 13 & 0.2044 \\
\hline IV & 10 & 6 & 4 & \\
\hline \multicolumn{5}{|l|}{ Residual tumor } \\
\hline$(+)$ & 15 & 6 & 9 & 0.9337 \\
\hline$(-)$ & 13 & 5 & 8 & \\
\hline \multicolumn{5}{|l|}{ First-line chemotherapy } \\
\hline CAP & 6 & 2 & 4 & 0.7362 \\
\hline CBDCA plus CPA & 9 & 2 & 7 & 0.3908 \\
\hline PTX plus CBDCA & 12 & 8 & 4 & 0.0294 \\
\hline \multicolumn{5}{|l|}{$\mathrm{COX}-2$} \\
\hline High & 18 & 7 & 11 & 0.9540 \\
\hline Low & 10 & 4 & 6 & \\
\hline
\end{tabular}

CAP, cyclophosphamide, doxorubicin and cisplatin; CBDCA, carboplatin; CPA, cyclophosphamide; PTX, paclitaxel.

Chemotherapy. All patients underwent 4-6 cycles of platinumbased systemic chemotherapy 2-3 weeks after primary surgery. Chemotherapy was according to the following three regimens: 6 patients $(21.4 \%)$ received cyclophosphamide $320 \mathrm{mg} / \mathrm{m}^{2}$, doxorubicin $30 \mathrm{mg} / \mathrm{m}^{2}$ and cisplatin $50 \mathrm{mg} / \mathrm{m}^{2}$ (CAP), 9 patients $(32.1 \%)$ received paraplatin $(\mathrm{AUC}=5)$ and cyclophosphamide $500 \mathrm{mg} / \mathrm{m}^{2}$ (CBDCA plus CPA), and 12 $(42.9 \%)$ were treated with paclitaxel $180 \mathrm{mg} / \mathrm{m}^{2}$ and paraplatin (AUC=5) (PTX plus CBDCA).

Cimetidine therapy. Cimetidine was offered to all patients with advanced ovarian cancer. The patients were informed that the effectiveness of the drug could not be guaranteed, but that it could be beneficial in treating the disease. After approval from the Gifu University Hospital Ethics Committee and informed patient consent in writing had been obtained, cimetidine was commenced and administered at a dose of $800 \mathrm{mg} /$ day two weeks before surgery to those patients who had given their informed consent. Cimetidine and chemotherapy were used synchronously for patients in the cimetidine group. The administration of such drugs continued for up to 2 years.

Immunohistochemistry. Tissue sections $(4-\mu \mathrm{m})$ fixed in $10 \%$ formalin and embedded in paraffin were mounted on poly-Llysin-coated slides (Sigma, St. Louis, MO). The sections were then deparaffinized, rehydrated and incubated for $30 \mathrm{~min}$ with $3 \% \mathrm{H}_{2} \mathrm{O}_{2}$ in methanol to quench endogenous peroxidase activity. After a short rinse in Tris-buffer, the sections were boiled in a microwave oven for 3-5 min in citrate buffer. Following rolling and rinsing in Tris-buffered saline, rabbit anti-COX-2 serum was applied to the sections for $20 \mathrm{~min}$ to block non-specific binding. The sections were then incubated overnight at $4{ }^{\circ} \mathrm{C}$ with monoclonal antibodies directed against COX-2. Antigen-antibody complexes were confirmed with the avidin-biotin-peroxidase complex (ABC) technique using a Vectastain $\mathrm{ABC}$ kit. Peroxidase activity was determined by 5 min incubation in 3,3'-diamino-benzidine tetrahydrochloride and $\mathrm{H}_{2} \mathrm{O}_{2}$ dissolved in citrate buffer.

The following monoclonal antibodies were purchased from Carman Inc. (Ann Arbor, MI). All tissue sections were evaluated by two different cytopathologists (K.N., H.M.) who had no prior knowledge of the clinical parameters. The immunoreactive score for COX-2 was calculated basically as described by Krajewska et al (13). Briefly, the intensity of immunostaining was assessed as follows: none, 0 ; weak, 1; moderate, 2; strong, 3. The percentage of positive tumor cells were as follows: none, $0 ;<10 \%, 1 ; 10-50 \%, 2 ; 51-80 \%, 3 ;>80 \%, 4$. For statistical analysis, the patients were divided into two groups based on their level of COX-2 expression $(<6$ or $>6)(1)$.

Clinical response and follow-up. Clinical data were obtained from the patients' records and follow-up data from the clinical registers. The patients were monitored every 3-4 


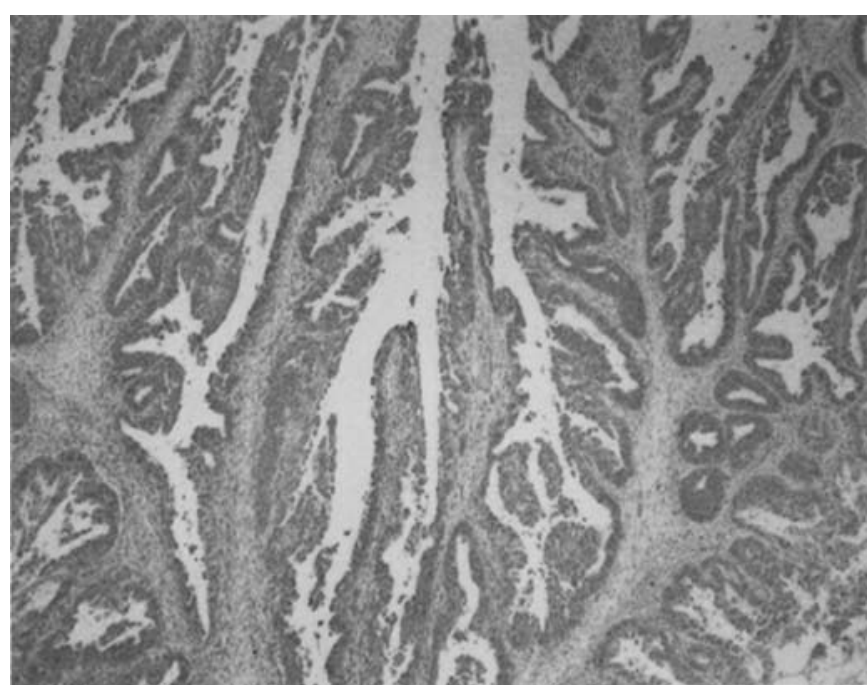

Figure 1. Immunohistochemical staining for COX-2 in a representative section of high-grade serous carcinoma. High COX-2 expression was restricted mainly to the tumor area (sABC x100).

A)

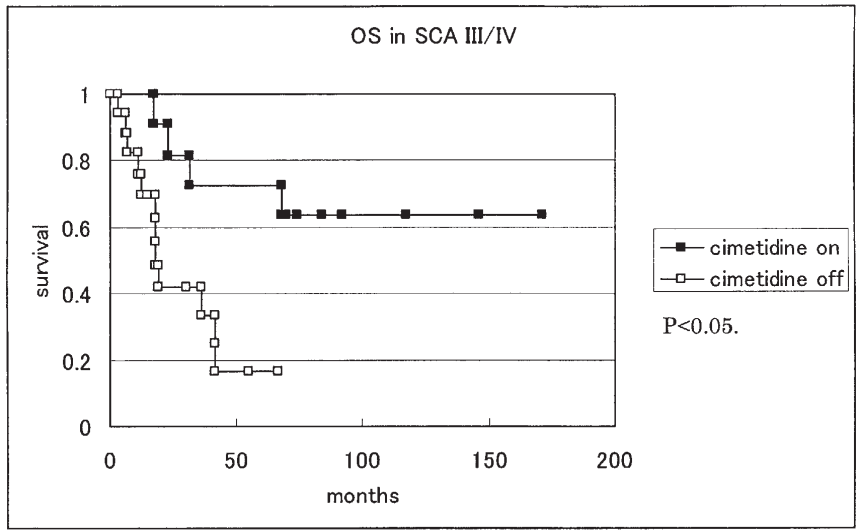

B)

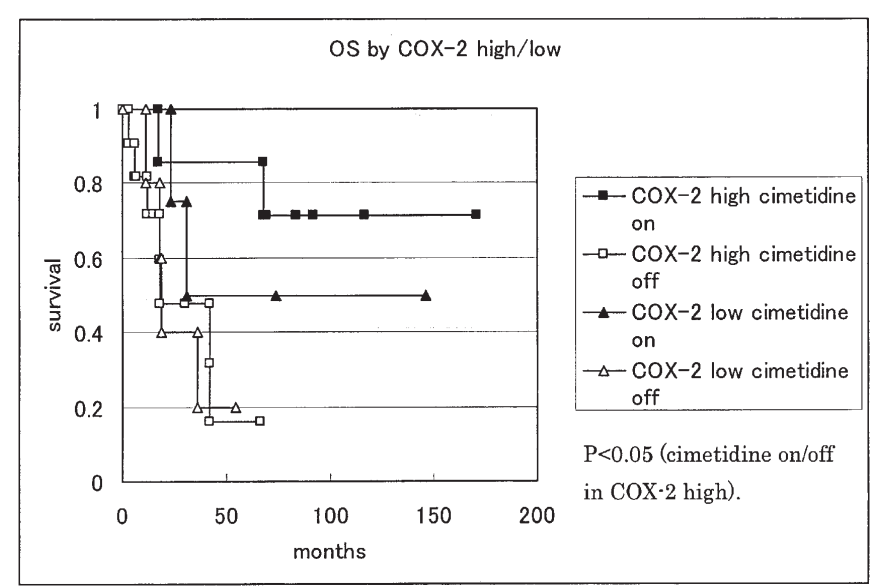

Figure 2. Kaplan-Meier curves for overall survival (OS) rates of patients with serous ovarian carcinoma in the cimetidine and control groups. (A) For patients with stage III/IV tumors, the OS of the cimetidine group was significantly longer than that of the control group $(\mathrm{P}<0.05)$. (B) For patients showing high expression of $\mathrm{COX}-2$, the $\mathrm{OS}$ rate of the cimetidine group was significantly higher than that of the control group $(\mathrm{P}<0.05)$ months during the first 3 years, and then every 6 months. In most cases of recurrence, the patients were treated with paclitaxel plus platinum-containing chemotherapy.

Survival analysis. Refractory free survival was defined as the period from the initial surgery to the time of recurrence or death, whichever occurred first. Overall survival (OS) was calculated from the date of the first surgery to the date of death or last contact. Medians and life tables were computed using the Kaplan-Meier method, and comparisons were made using the log-rank test.

\section{Results}

Follow-up data were available for 28 patients. As of December 2006 , the median follow-up period was $3.1 \pm 1.4$ years. The mean survival rate was $3.4 \pm 3.5$ years and $50 \%$ survival was 18.0 months. As for first-line chemotherapy, no significant differences were found between the three regimens (data not shown).

The background ratios, including histological grades, residual tumors and first-line chemotherapy between the cimetidine and control groups showed no differences.

Fig. 1 shows immunohistochemical staining for COX-2 in a representative section of high-grade serous carcinoma. Immunohistochemical expression scores were $7.1 \pm 2.9$ for the cimetidine group versus $7.5 \pm 2.8$ for the control group. There were no differences in COX-2 score between the cimetidine and control groups $(\mathrm{P}=0.5476)$.

The OS rate of the cimetidine group was significantly higher than that of the control group. The cimetidine group $(\mathrm{P}<0.05)$ had a significantly better prognosis than the control group (Fig. 2A). In patients showing high expression of COX-2, the OS rate of the cimetidine group was significantly higher than that of the control group (Fig. 2B, P<0.05).

\section{Discussion}

In the present study, platinum-based chemotherapy combined with cimetidine significantly improved the survival as well as the recurrence rate of patients with advanced stage (FIGO stage III/IV) serous ovarian cancer. These results suggest that cimetidine contributes to an improved prognosis for such patients.

In patients with colorectal or ovarian carcinomas, overexpression of COX-2 is thought to be associated with resistance to chemotherapy $(7,8)$. On the other hand, cimetidine has been reported to inhibit histamine-induced angiogenesis (11) as well as epidermal growth factor-induced cell signaling (14). Cimetidine is also reported to induce apoptosis of human salivatory gland tumor cells (15). Notably, cimetidine improved the survival of patients whose ovarian cancer overexpressed COX-2. This is the first report of cimetidine and systemic chemotherapy improving the survival of ovarian cancer patients.

Although the number of patients administered cimetidine was relatively small, our findings suggest that adding this drug to systemic chemotherapy for patients with advanced ovarian cancer may prolong their survival. A randomized controlled study is needed to further confirm these findings. 


\section{References}

1. Ali-Fehmi R, Che M, Khalifeh I, Malone JM, et al: The effect of cycloxgenase- 2 expression on tumor vascularity in advanced stage ovarian serous carcinoma. Cancer 98: 1423-1429, 2003.

2. Tamakoshi K, Kondo T, Yasuya H, Hori Y, Kikkawa F and Toyoshima H: Trends in the mortality (1950-1970) and incidence (1975-1993) of malignant ovarian neoplasm among Japanese women: analses by age, time, and birth cohort. Gynecol Oncol 83: 64-71, 2001.

3. Warwick J, Kehoe S, Earl H, Luesley D, Redman C and Chan KK: Long-term follow-up of patients with advanced ovarian cancer treated in randomized clinical trials. Br J Cancer 72: $1513-1517,1995$

4. The international Collaborative Ovarian Neoplasm Group: Paclitaxel plus carboplatin versus standard chemotherapy with either single-agent carboplatin or cyclophosphamide, doxorubicin, and cisplatin in women with ovarian cancer. The ICON3 randomised trial. Lancet 360: 505-515, 2002.

5. Kikuchi Y, Oomori K, Kizawa I and Kato K: Effects of cimetidine on tumor growth and immune function in nude mice bearing human ovarian carcinoma. J Natl Cancer Inst 74: 495-498, 1985.

6. Matsumoto S: Cimetidine and survival with colorectal cancer. Lancet 346: 115, 1995.

7. Cianchi F, Cortesini C, Schiavone N, et al: The role of cyclooxygenase- 2 in mediating the effects of histamine on cell proliferation and vascular endothelial growth factor production in colorectal cancer. Clin Cancer Res 11: 6807-6815, 2005.
8. Raspollini MR, Amunni G, Villanucci A, Boddi V and Taddei GL: Increased cyclooxygenase-2 (COX-2) and P-gycoproein-170 (MDR1) expression is associated with chemotherapy resistance and poor prognosis. Analysis in ovarian carcinoma patients with low and high survival. Int J Gynecol Cancer 15: 255-260, 2005.

9. Ferrandina G, Lauriola L, Zannoni GF, et al: Increased cyclooxygenase-2 (COX-2) expression is associated with chemotherapy resistance and outcome in ovarian cancer patient. Ann Oncol 13: 1205-1211, 2002.

10. Kobayashi K, Matsumoto S, Morishima T, Kawabe T and Okamoto T: Cimetidine inhibits cancer cell adhesion to endothelial cells and prevents metastasis by blocking E-selectin expression. Cancer Res 60: 3978-3984, 2000.

11. Ghosh AK: Regulation by prostaglandin E2 and histamine of angiogenesis in inflammatory granulation tissue. Yakugaku Zasshi 123: 295-303, 2003

12. Serov SF, Scully RE and Sobin LH: Histological typing of ovarian tumours. In: International Histological Classification of Tumors. WHO, Geneva, pp37-59, 1973.

13. Krajewska M, Fenoglio-Preiser CM, Krajewski S, et al: Immunohistochemical analysis of Bcl-2 family proteins in adenocarcinoma of the stomach. Am J Pathol 149: 1449-1457, 1996.

14. Fujikawa T, Shiraha H, Nakanishi Y, et al: Cimetidine inhibits epidermal growth factor-induced cell signaling, J Gastroenterol Hepatol 22: 436-443, 2007.

15. Fukuda M, Tanaka S, Suzuki S, Kusama K, Kaneko T and Sakashita H: Cimetidine induced apoptosis of human salivary gland tumor cells. Oncol Rep 17: 673-678, 2007. 